\title{
Wave-Packet Calculation of Sharp-Energy $S$-Matrix Elements for a Three-Body System in the Breakup Regime
}

\author{
Z. C. Kuruoglu \\ Chemistry Department, Bilkent University, Ankara, Turkey \\ F. S. Levin \\ Physics Department, Brown University, Providence, Rhode Island 02912 \\ (Received 31 August 1989)
}

\begin{abstract}
Sharp-energy $S$-matrix elements have been successfully extracted from a wave-packet description of three-boson and quartet-spin, neutron-deuteron scattering at energies well above the breakup threshold. Essential to the success of this procedure is use of an expansion basis comprising functions from each of the three two-cluster arrangements.

PACS numbers: $03.65 . \mathrm{Nk}, 25.10 .+\mathrm{s}$
\end{abstract}

Although the time-dependent Schrödinger equation provides the most natural framework for describing quantal collision phenomena, ${ }^{\prime}$ its use in numerical analysis has lagged well behind that of time-independent methods. Two factors underlie this. One is a belief that the time-dependent (TD) approach is too computationally expressive due to the required time integration. The other is the conviction-still widespread at least in the light-ion and few-nucleon nuclear physics community despite the work, e.g., of Ref. 2-that sharp energy or momentum values of collision amplitudes cannot be extracted numerically because of the averaging used in defining the relevant wave packets. Recent developments, however, have made it clear that there is no longer any foundation for these beliefs.

One of these developments is a significant advance in computational technology and the attendant numerical strategies and algorithms. This has made TD methods not only computationally feasible, but also competitive with time-independent (TI) methods in some cases. The other development, largely the effort of workers in the area of atom-molecule reactive collisions, ${ }^{2}$ has been the demonstration that via a scattering-theoretic result, ${ }^{\prime}$ sharp-energy and -momentum values of $S$-matrix elements can be extracted directly from a time-dependent Schrödinger equation analysis.

Relatively few of these latter computations have yet been carried out, and almost all are for energies below the threshold for breakup of an initial two-body collision system into final states containing three (or more) bodies. Thus, the influence of breakup on the extraction of sharp $S$-matrix elements in the TD wave-packet approach has not been studied in any detail. ${ }^{3}$ As a result, the most interesting sector of the three-nucleon collision system has not been investigated from this most fundamental of descriptions. In work begun several years ago and recently completed, however, we have closed this particular gap; the present Letter describes some of our procedures, results, and conclusions.
Numerical solution of the TD problem means that one uses an approximation space, defined, e.g., by specifying a set of basis functions, or equivalently but somewhat implicitly, by introducing a finite domain for the spatial variables (positions or momenta) and then discretizing this domain, as in the finite-difference, fast-Fouriertransform, or finite-element methods. We worked in momentum space, so that potentials became integral operators; this was easier for us to handle than the differential operators occurring in a coordinate representation. In addition, the support of the momentum-space wave packets is constant: The packets do not spread. The momentum-space domain was partitioned into finite elements and piecewise quadratics were used as the basis functions.

Because rearrangement and breakup can occur in a three-particle system, the problem of different sets of coordinates and the existence of various asymptotic Hamiltonians made the choice of approximation space very nontrivial. That is, a limited set of basis functions in the Jacobi momenta of one arrangement cannot accurately describe configurations involving other arrangements. In order to avoid excessively large bases yet still achieve stable, converged results, it was absolutely essential that our approximation space was constructed as the union of the arrangement-channel approximation spaces. Hence, our expansion basis is nonorthogonal, consisting of functions that depend on each of the three sets of two-fragment Jacobi momenta. This is akin to the coupled reaction channel (CRC) Ansatz familiar from the TI approach. ${ }^{4}$ Our procedure differs from the conventional finite-element method $^{5}$ in that discretization was not done in a single set of variables.

The Hamiltonian of our three-particle system is $H=H_{0}+V_{12}+V_{13}+V_{23}$, where $H_{0}$ is the sum of the two kinetic-energy operators and $V_{\beta \gamma} \equiv V_{\alpha}$ is the (shortranged) interaction between the pair of particles $\beta \gamma . H_{0}$ can be expressed as $H_{0}=k_{\alpha}+K_{\alpha}, \alpha=1,2,3$, with $k_{\alpha}$ being the relative motion kinetic-energy operator for the 
pair $\beta \gamma$ and $K_{\alpha}$ being the kinetic-energy operator for the motion of particle $\alpha$ relative to the c.m. of the pair $\beta \gamma$. In terms of the Jacobi momenta $\mathbf{q}_{a}$ and $\mathbf{p}_{a}$, the eigenstates of $K_{\alpha}$ and $k_{\alpha}$ are denoted $\left|\mathbf{q}_{\alpha}\right\rangle$ and $\left|\mathbf{p}_{\alpha}\right\rangle$, respectively; their energies are $E_{q_{a}}$ and $E_{p_{a}}$. The full $H$ can be decomposed into three different asymptotic or arrangement-channel Hamiltonians $H_{a}$ and corresponding channel interactions $V^{\alpha}: H_{\alpha}=K_{\alpha}+k_{\alpha}+V_{\alpha}, V^{\alpha}=V_{\beta}$ $+V_{\gamma}, \alpha=1,2,3$. Here, $h_{\alpha}=k_{\alpha}+V_{\alpha}$ is the barycentric Hamiltonian for the pair $\beta \gamma$ and is assured to support at least one bound state $\left|\psi_{\alpha n}\right\rangle$, i.e., $h_{\alpha}\left|\psi_{\alpha n}\right\rangle=E_{\alpha n}\left|\psi_{a n}\right\rangle$. Hence the (non-normalizable) asymptotic states in channel $\alpha$ are the products $\left|\psi_{\alpha n} \mathbf{q}_{\alpha}\right\rangle$ with energy $E_{a n q_{\alpha}}=E_{\alpha n}$ $+E_{q_{a}}$. We also define the TD channel states via

$$
\begin{aligned}
\left|\psi_{a n}(t) q_{\alpha}(t)\right\rangle & =\exp \left(-i H_{a} t\right)\left|\psi_{a n} \mathbf{q}_{\alpha}\right\rangle \\
& =\left|\psi_{a n} \mathbf{q}_{\alpha}\right\rangle \exp \left(-i E_{a n q_{a}} t\right) .
\end{aligned}
$$

Finally, the position vectors conjugate to $\mathbf{p}_{\alpha}$ and $\mathbf{q}_{\alpha}$ are denoted $\mathbf{x}_{\alpha}$ and $\mathbf{y}_{\alpha}$, respectively.

To describe the collision generated by $\alpha$ incident on the pair $\beta \gamma$, we take the initial $(t=0)$ wave packet to be

$$
\left|\Phi_{a n}(0)\right\rangle=\left|\psi_{a n}\right\rangle\left|f_{a \mathbf{q}_{0}}\right\rangle,
$$

where $\left|f_{a \mathbf{q}_{0}}\right\rangle=\int d^{3} q_{a} A_{\mathbf{q}_{0}}\left(\mathbf{q}_{\alpha}\right)\left|\mathbf{q}_{\alpha}\right\rangle$, with $A_{\mathbf{q}_{0}}\left(\mathbf{q}_{\alpha}\right)$ chosen so that $\left|f_{\alpha \mathbf{q}_{0}}\right\rangle$ is a packet with average momentum $\mathbf{q}_{0}$ and average position $\mathbf{y}_{a}$. The initial position $\mathbf{y}_{0}$ is chosen well outside the range of $V^{\alpha}$. The exact time evolution of $\left|\Phi_{a n}(0)\right\rangle$ is given by

$$
\left|\Phi_{a n}(t)\right\rangle=\left|\psi_{\alpha n}(t)\right\rangle\left|f_{a \mathbf{q}_{0}}(t)\right\rangle,
$$

where

$$
\left|f_{\alpha \mathbf{q}_{0}}(t)\right\rangle=\int d^{3} q_{\alpha} A_{\mathbf{q}_{0}}\left(\mathbf{q}_{\alpha}\right)\left|\mathbf{q}_{\alpha}\right\rangle \exp \left(-i E_{q} t\right) .
$$

Although standard choices of $A_{\mathbf{q}_{0}}\left(\mathbf{q}_{\alpha}\right)$ allow $\left|\Phi_{\alpha n}(t)\right\rangle$ to be evaluated analytically, we need and work with the numerically propagated free wave packet $\left|\tilde{\Phi}_{a n}(t)\right\rangle$ $=\left|\tilde{\psi}_{a n}(t)\right\rangle\left|\tilde{f}_{q_{a}}(t)\right\rangle$, the tilde indicating the numerical time evolution.

The exact and numerical solutions to the TD Schrödinger equation that evolve from $\left|\Phi_{a n}(0)\right\rangle$ are denoted $|\Psi(t)\rangle$ and $|\tilde{\Psi}(t)\rangle$, respectively. A fundamental result of TD scattering theory is that ${ }^{1}$

$$
\lim _{t \rightarrow \infty}\left\{P_{\beta}|\Psi(t)\rangle=P_{\beta} S_{\beta \alpha}\left|\Phi_{\alpha}(t)\right\rangle\right\},
$$

where $S_{\beta a}$ is the $\beta \alpha$ element of the usual scattering or $S$ operator and $P_{\beta}$ is the projector onto the asymptotic states of channel $\beta$, viz.,

$$
P_{\beta}=\sum_{n} \int d^{3} q_{\beta}\left|\psi_{\beta n} \mathbf{q}_{\beta}\right\rangle\left\langle\psi_{\beta n} \mathbf{q}_{\beta}\right| .
$$

(Note that $P_{\beta}$ could be made time dependent.) Numerically, (2) is replaced by

$$
\tilde{P}_{\beta}(T)|\Psi(T)\rangle=\tilde{P}_{\beta}(T) S_{\beta \alpha}\left|\tilde{\psi}_{\alpha n}(T) \tilde{f}_{\alpha \mathbf{q}_{0}}(T)\right\rangle,
$$

where $T \gg 0$ and $\left|\tilde{\psi}_{a n}(T)\right\rangle$ is the numerically propagated bound state, used to construct $\tilde{P}_{\beta}(T)$, now necessarily time dependent.

Equation (3) implies an integration over momenta. To extract a sharp-energy $S$-matrix element, first the energy-conserving property of $S_{\beta \alpha}$ is used, viz.,

$$
\begin{aligned}
\left\langle\psi_{\beta m} \mathbf{q}_{\beta}\left|S_{\beta \alpha}\right| \psi_{\alpha n} \mathbf{q}_{n}\right\rangle & \\
& =S_{\beta m, a n}\left(\hat{q}_{\beta} \leftarrow \hat{q}_{\alpha} ; E\right) \delta\left(E_{\beta m}+E_{q \beta}-E_{\alpha n}-E_{q \alpha}\right),
\end{aligned}
$$

$E=E_{\alpha n}+E_{q \alpha}$, and then an angular momentum decomposition is made so that the angular integration in (3) can also be carried out. Equation (3) then becomes, in schematic form,

$$
\begin{aligned}
& N_{\beta \alpha}\left[S_{\beta m, a n}(E)\right]_{\text {partial wave }} \\
& =\frac{\left[\left\langle\tilde{\psi}_{\beta m}(T) \mathbf{q}_{\beta}(T) \mid \tilde{\Psi}(T)\right\rangle\right]_{\text {partial wave }}}{\left[\left\langle\mathbf{q}_{\alpha}(T) \mid \tilde{f}_{\alpha \mathbf{q}_{0}}(T)\right\rangle\right]_{\text {partial wave }}},
\end{aligned}
$$

where $N_{\beta \alpha}$ is a kinematic factor depending only on the masses of the particles and "partial wave" refers to the total angular momentum representation.

It has already been established that (4) yields reliable results for energies below the breakup threshold. ${ }^{2}$ In our test of (4) above the breakup threshold, we have taken the pair interactions to be separable $S$-wave potentials with standard Yamaguchi form factors, ${ }^{6}$ e.g., $\left\langle\mathbf{p}_{\alpha}\left|V_{\alpha}\right| \mathbf{p}_{\alpha}^{\prime}\right\rangle=\lambda\left\langle\mathbf{p}_{\alpha} \mid \chi_{\alpha}\right\rangle\left\langle\chi_{\alpha} \mid \mathbf{p}_{\alpha}^{\prime}\right\rangle, \quad\left\langle\mathbf{p}_{\alpha} \mid \chi_{\alpha}\right\rangle=\left(p_{\alpha}^{2}+\beta_{\alpha}^{2}\right)^{-1}$. Two versions of our model were used: three identical, spinless bosons and the quartet neutron-deuteron system. Each is an example of the Amado-Mitra model. ${ }^{7}$ Only $S$-wave $(L=0)$ states of relative motion between each particle $\alpha$ and the concomitant pair $\beta \gamma$ were considered, since higher $L$ values add complexity but not a better test of the basic method. $A_{\mathbf{q}_{0}}\left(\mathbf{q}_{\alpha}\right)$ was chosen to be a Gaussian.

To form the approximation space, cutoff values $q_{a, \max }$ and $p_{\alpha, \max }$ were chosen, based on the behavior of the $V_{\alpha}$ and the value of $q_{0}$. For each $\alpha$, the finite intervals $\left[0, q_{a, \max }\right]$ and $\left[0, p_{a, \max }\right]$ were divided into subintervals (finite elements). Based on this discretization, local interpolation bases $\left\{u_{\alpha i}\left(p_{\alpha}\right)\right\}_{i}^{N_{a}}{ }_{1}$ and $\left\{v_{\alpha j}\left(q_{\alpha}\right)\right\}_{j=1}^{M_{a}}$ were introduced for each $\alpha$. The direct products of piecewise quadratics $\left|u_{a i} v_{a j}\right\rangle$ form the finite-element basis for arrangement $\alpha$. The full approximation space is spanned by the union of the three arrangement bases, an entity that was essential to our achieving successful results.

The full wave packet was thus expanded as

$$
|\tilde{\Psi}(t)\rangle=\sum_{\beta=1}^{3} \sum_{i, j} C_{\beta i j}(t)\left|u_{\beta i} v_{\beta j}\right\rangle ;
$$

this leads to

$$
\mathbf{S}^{-1} \mathbf{H C}(t)=i \dot{\mathbf{C}}(t),
$$

in matrix notation in which $\mathbf{S}$ is the overlap matrix of basis functions, $\mathbf{H}$ is the Hamiltonian matrix, and $\mathbf{C}$ is 
the column vector of unknown coefficients. A similar result describes the propagation of the free wave packet in each arrangement $\alpha$.

One might consider solving (5) directly via $\mathbf{C}(t)$ $=\exp \left\{-i \mathbf{S}^{-1} \mathbf{H} t\right\} \mathbf{C}(0)$ or even constructing the $S$ matrix directly ${ }^{3}$ from

$$
\tilde{S}_{\beta \alpha}=\exp \left\{i \mathbf{S}_{\beta}^{-1} \mathbf{H}_{\beta} T\right\} \exp \left\{-i \mathbf{S}^{-1} \mathbf{H} T\right\},
$$

where $\mathbf{S}_{\beta}$ and $\mathbf{H}_{\beta}$ are the channel $\beta$ analogs of $\mathbf{S}$ and $\mathbf{H}$. We did not do so for several reasons. First, exponentiation of (especially non-Hermitian) matrices is quite nontrivial. Second, how to extract the energy-conserving $\delta$ function is not apparent. Third, choosing the value of $T$ in the $\tilde{S}_{\beta \alpha}$ formula is problematic, since the formula is equivalent to using $N$ initial conditions such that the initial wave packets are the $\left\{\left|u_{\alpha i} v_{\alpha i}\right\rangle\right\}$. But these basis functions are standing, not purely incoming, waves. This gives rise to severe boundary reflection problems.

In order to avoid these difficulties, we proceeded instead by using the small-time, finite-difference approximation to (5), viz., ${ }^{8}$

$$
\mathbf{C}\left(t_{n+1}\right)=\mathbf{C}\left(t_{n-1}\right)-2 i \Delta t \mathbf{S}^{-1} \mathbf{H C}\left(t_{n}\right),
$$

where $\Delta t$ is the time interval and $t_{n}=n \Delta t$. Equation (6) is probably the simplest conditionally stable, explicit propagation scheme and is especially well suited for implementation on vector computers since the basic steps are repeated matrix-vector operations followed by vector-vector addition. More sophisticated algorithms could be used, but time propagation was never a problem in our computations, especially with use of the numerically propagated free wave packets: Any inaccuracies introduced by numerical treatment of the $H_{\alpha}$ dynamics are canceled when (4) is used to extract $S$-matrix elements. That is, $\tilde{\Psi}_{\alpha}(t) / \tilde{\Phi}_{\alpha}(t)$ yielded accurate $S$-matrix elements even though $\tilde{\Phi}_{\alpha}(t)$ and $\Phi_{\alpha}(t)$ differed in phase (but only very slightly in magnitude).

The restrictions $p_{\alpha} \leq p_{\alpha, \max }$ and $q_{\alpha} \leq q_{\alpha, \max }$ and the introduction of a finite basis over the truncated domains means that in coordinate space the system is effectively enclosed in a box as well as represented by a finite basis. The support of the coordinate representation is determined by the size of the basis or the fineness of the discretization in momentum space. Care must be (and was) taken to ensure avoidance of spurious effects resulting from reflections at the (implicit) coordinate-space boundaries. In this respect, low-energy collisions can lead to problems because the fast-moving components in the wave packet may reach the boundaries before the slow-moving components leave the interaction region. As $q_{0}$ decreases and passage times increase, more momentum-space basis functions or equivalently finer meshes are needed. Using 20- to 45-point discretization in $q_{\alpha}$ and $p_{\alpha}$, the smallest $q_{0}$ for our model which gave reliable results was $q_{0} \cong 1.0 \mathrm{fm}^{-1}$, corresponding to a bombarding energy of about $30 \mathrm{MeV}$. The basis sizes used (see below) were small compared, e.g., to the number of mesh points used in coordinate-space fastFourier-transform methods, typically ${ }^{2} 256$. And, to stress this point once more, it is the CRC-type expansion Ansatz that allowed us to achieve success for $q_{0}$ as low as $1.0 \mathrm{fm}^{-1}$ while keeping the matrix dimensions reasonable (around 2600 for $q_{0}=1.0 \mathrm{fm}^{-1}$ ).

Some results for the lowest $q_{0}$ value $\left(1 \mathrm{fm}^{-1}\right)$ in the model of quartet-spin, neutron-deuteron scattering are shown in Table I, which lists $\operatorname{Re} S_{\text {el }}, \operatorname{Im} S_{\text {el }}$, and the probability that elastic scattering occurs for various $q$ in the wave packet and for sufficiently large sampling times $T$ that the packet is outside the interaction region and outgoing. The average values of $y_{\alpha}$ in the projected wave packet, $\tilde{P}_{\alpha}(T)|\tilde{\Psi}(T)\rangle$, are $20.3 \mathrm{fm}$ for $T=18$, and 58.8 fm for $T=45$. $T=45$ in the present case is nearly maximal: Not much beyond it, reflections begin to occur. The exact results in Table I were obtained by numerically solving the Faddeev integral equations using a solution method based on the Schwinger variational principle, and are accurate to at least three significant figures.

Since the pair potentials each support only one bound state (at 2.2 MeV), the spin-isospin structure of this system leads to the following elastic $S$-matrix element: $S_{\text {el }}=S_{11}-0.5\left(S_{21}+S_{31}\right)$. The numbers were obtained using $17 p$-mesh points ranging from 0 to $7.6 \mathrm{fm}^{-1}$ (31 basis functions) and $44 q$-mesh points ranging from 0 to $5.6 \mathrm{fm}^{-1}$ ( 85 basis functions), and are reliable to 1 part in $10^{3}$. The values $\Delta t=0.003$ and $t_{\max }=48.6$ time units were used ( $\left.\hbar=M_{p}=1\right)$. All wave-packet normalizations were found to differ from unity by less than 0.01 at all $T$ sampled in the program. The accuracy of these results for $q_{0}=1 \mathrm{fm}^{-1}$ ( $\approx 30 \mathrm{MeV}$ ) is typical of those obtained at higher $q_{0}{ }^{9}$ In addition, equally good results have been obtained in the identical boson case. ${ }^{9}$

We have established in this Letter that sharp-momentum and -energy values of $S$-matrix elements can be extracted from a time-dependent, wave-packet analysis of

TABLE I. Selected $S_{\mathrm{el}}(q)$ values, quartet $n+d$ case; $q_{0}=1$ $\mathrm{fm}^{-1}$. EP denotes probability that elastic scattering occurs and WP denotes wave packet.

\begin{tabular}{llrrr}
\hline \hline$q$ & & Exact & WP, $T=18$ & WP, $T=45$ \\
\hline 0.8 & $\operatorname{Re} S_{\text {el }}$ & -0.234 & -0.240 & -0.233 \\
& $\operatorname{Im} S_{\text {el }}$ & 0.881 & 0.912 & 0.897 \\
& $\operatorname{EP}$ & 0.834 & 0.889 & 0.859 \\
1.0 & $\operatorname{Re} S_{\text {el }}$ & 0.153 & 0.157 & 0.158 \\
& $\operatorname{Im} S_{\text {el }}$ & 0.869 & 0.849 & 0.872 \\
& $\operatorname{EP}$ & 0.778 & 0.746 & 0.785 \\
1.2 & $\operatorname{Re} S_{\text {el }}$ & 0.441 & 0.436 & 0.434 \\
& $\operatorname{Im} S_{\text {el }}$ & 0.762 & 0.759 & 0.785 \\
& $\operatorname{EP}$ & 0.775 & 0.766 & 0.779 \\
& $\operatorname{Re} S_{\text {el }}$ & 0.768 & 0.785 & 0.753 \\
& $\operatorname{Im} S_{\text {el }}$ & 0.502 & 0.498 & 0.499 \\
& $\operatorname{EP}$ & 0.842 & 0.865 & 0.815 \\
\hline \hline
\end{tabular}


three-particle collisions for energies well above the breakup threshold. The best accuracy achieved so far for the lower range of $q$ values with respect to $q_{0}$ is an error of about $1 \%$; these can probably be improved somewhat by one or more of more careful placing of the nodes, using other propagation methods, or using higher-order interpolates. Study of these and application of the method to other systems is currently in progress.

This work has been supported in part by research grants from the U.S. Department of Energy and the North Atlantic Treaty Organization. One of us (Z.C.K.) acknowledges hospitality, computing facilities, and financial support from the following institutions, where portions of these investigations were carried out: Tubitak Research Institute, University of Iowa, George Washington University, and University of New Mexico (where the U.S. National Science Foundation provided partial support).

'See, e.g., J. R. Taylor, Scattering Theory (Wiley, New York, 1972); R. G. Newton, Scattering Theory of Waves and Particles (Springer-Verlag, New York, 1982), 2nd ed.

${ }^{2}$ See, e.g., Y. Sun, R. C. Mowrey, and D. J. Kouri, J. Chem. Phys. 87, 339 (1987), and references cited therein. For refer- ences to the older chemical literature on this subject, see, e.g., V. Mohan and N. Sathamurthy, Comput. Phys. Rep. 7, 214 (1988). A very different method than that of the present manuscript for extracting sharp-energy $S$-matrix elements, but in the single-rearrangement-channel, nucleon-nucleon case, is given by J. Holz and W. Glöckle, Phys. Rev. C 37, 1386 (1988).

${ }^{3}$ A non-wave-packet method of directly constructing the $S$ operator by means of strong-operator approximations has been introduced by H. Kröger, e.g., J. Math. Phys. 26, 970 (1985) and Phys. Rev. C 31, 1118 (1985), and references cited therein.

${ }^{4}$ For references to the CRC method, see, e.g., T. Ohmura, B. Imanishi, M. Ichimura, and M. Kawai, Prog. Theor. Phys. 44, 1242 (1970), and S. R. Cotanch and C. M. Vincent, Phys. Rev. C 14, 1739 (1976), and references cited therein.

${ }^{5}$ See, e.g., P. M. Prenter, Splines and Variational Methods (Wiley, New York, 1975).

${ }^{6}$ Y. Yamaguchi, Phys. Rev. 95, 1628 (1954).

${ }^{7}$ For references to the Amado-Mitra model, see R. D. Amado, in Three-Particle Scattering in Quantum Mechanics, edited by J. Gillespie and J. Nuttall (Benjamin, New York, 1968), and A. N. Mitra, in Advances in Nuclear Physics, edited by M. Baranger and E. Vogt (Plenum, New York, 1969), Vol. III.

${ }^{8}$ A. Askar and A. S. Cakmak, J. Chem. Phys. 68, 2794 (1978).

${ }^{9}$ This point will be discussed in detail in articles in preparation. 